\title{
Aplicação de Tratamento Estatístico Utilizando o Método de Tukey para Análise de Ruído e Vibração de Veículos SUV
}

\author{
Lays E. Cunha, Paulo Zavala \& Fábio S. Gomes
}

A aplicação de métodos combinados para análise estatística de parâmetros de conformidade é convencional e utilizado desde os primeiros conceitos de estatística descritiva. Partindo deste princípio, este trabalho teve como objetivo demonstrar o comportamento de uma proposta de métricas para ruído e vibração interna veicular, utilizando para tanto a análise três sigma (distribuição normal) e o método de John Tukey. Este método em conjunto com os resultados da análise sigma caracterizou valores de distintas grandezas, possibilitando padronizar para os veículos da mesma categoria (SUV) os limites adotados. Os resultados obtidos na amostra mensurada geraram valores de LMS (limite superior) advindos do método de tukey, estes correlacionaram se com a amplitude estudentizada possibilitando a adaptação de um padrão e viabilizando confrontar as medições subsequentes destinadas a avaliação de conformidade produtiva.

Palavras-chave: ruído; vibração; método de tukey.

The application of combined methods for statistical analysis of compliance parameters is conventional and used since the first concepts of descriptive statistics. Based on this principle, this work aimed to demonstrate the behavior of a proposal of metrics for noise and vehicular internal vibration, using the three sigma analysis (normal distribution) and the John Tukey method. This method together with the results of the sigma analysis characterized values of different quantities, making it possible to standardize the adopted limits for vehicles of the same category (SUV). The results obtained in the measured sample generated LMS values (upper limit) from the tukey method, which correlated with the studied amplitude, allowing the adaptation of a standard and enabling the comparison of subsequent measurements aimed at evaluating productive conformity.

Keywords: noise; vibration; tukey's method. 


\section{Introdução}

Por vários anos, pesquisadores têm buscado definir o sentido da utilização dos conceitos estatísticos no cotidiano. A utilização de teorias baseadas em probabilidade, a estatística possibilita-nos fazer inferências de fenômenos futuros, com base em uma coleta de dados de eventos passados, trabalhando tanto com dados observacionais quanto em experimentos, minimizando assim a incerteza dos experimentos. Para profissionais da engenharia ela proporciona previsões úteis sobre a demanda e distribuição de eventos na área de planejamento e controle de produção ${ }^{1}$.

Neste raciocínio Toledo ${ }^{2}$ descreve a estatística como: Cada vez mais acentuada em qualquer atividade profissional da vida moderna. Nos seus mais diversificados ramos de atuação, as pessoas estão frequentemente expostas à Estatística, utilizando-a com maior ou menor intensidade. Isto se deve às múltiplas aplicações que o método estatístico proporciona àqueles que dele necessitam.

Portanto, de acordo com as considerações apresentadas acima, o controle estatístico de processos é uma ferramenta baseada em conceitos e técnicas da estatística e da engenharia de produção que trabalha em conjunto no controle da qualidade nas etapas de um processo, ou seja, é um método preventivo a fim de comparar resultados com um padrão já existente e a partir de técnicas estatísticas eliminar ou controlar certas variações ${ }^{3}$. O Controle de Qualidade também é uma medida adotada com o objetivo de definir e monitorar padrões de produto e processo produtivo almejados, visando elevar o grau de satisfação do consumidor, acionistas, funcionários e fornecedores ${ }^{4}$.

Seguindo este pensamento existem alguns marcos históricos que demonstram as ocorrências e eventos desde a criação da estatística até o desenvolvimento do método de Tukey em $1953^{5}$.

- 5000 aC - Registros egípcios de presos de guerra.

- 695 - Primeira utilização da média ponderada pelos árabes na contagem de moedas.
- 1918 - Definição formal de Variância em um artigo de Genética (Fisher) e Distribuição de von Mises.

- 1923 - Tabela A NOVA (Fisher) e Processo de Wiener.

- 1930 - Controle de Qualidade nas indústrias, Inferência Fiducial (Fisher), Distância de Mahalanobis, Tempo Médio de Espera na Fila M/G/1 (Pollaczek) e Fundação da Econometria.

- 1949 - Método de Linearização, Eficiência em Grandes Amostras (Neyman) e Teste de Aditividade de Tukey.

- 1953-Teste de Tukey da diferença honestamente significativa.

Ao realizar a análise de variância (ANOVA) de um experimento com dois tratamentos, visualiza-se apenas pela média qual o melhor tratamento. Entretanto, quando existe mais de dois tratamentos, fazendo apenas o teste de " $\mathrm{f}$ " (teste que aponta a existência de diferença entre as médias dos tratamentos) não há indicação precisa de qual o melhor tratamento. Neste caso, é necessário aplicar um teste de comparação de médias dos tratamentos, daí podendo concluir qual o melhor tratamento . $^{5}$

Então os testes de comparação de média servem como um complemento para o estudo da análise de variância. Há vários testes de comparação de médias, entre os quais podem ser citados: teste de Tukey, teste de Duncan, teste de Scheffé, teste de Dunnet e teste de Bonferroni ${ }^{5}$.

Dito isto, visando melhorar o controle estatístico de processos (CEP) para limiares produtivos de ruído interno, há a necessidade de compreender o ramo da engenharia destinado ao estudo e avaliação de fenômenos acústicos e oscilatórios. Este é representado pela sigla NVH (Noise, vibration and Harshness / Ruído, vibração e aspereza). Esta sigla tem um significado confuso, porém pode ser definida como a qualidade acústica levando em consideração as exigências e o grau de satisfação que o corpo humano requer ${ }^{7}$. A qualidade acústica tem três tipos distintos de influências variáveis: Psicológicas (avaliação), Psicoacusticas (percepção) e físicas (Intensidade). AbdelRahman considera que mensurar a qualidade acústica 
de um veículo, além de multidimensional (culminando na classificação de experiências sensoriais humanas) é uma tarefa subjetiva ${ }^{8}$. A identidade de um veículo também está expressa nas características acústicas dele, tais parâmetros influenciam significativamente na decisão do cliente ${ }^{9}$.

A estatística tem sido aliada da acústica, pois o trabalho em conjunto delas trouxe uma parametrização dos dados exigidos normativamente para apresentação de controle de ruídos. Ações de estudos comparativos entre marcas e modelos têm sido amplamente feitas por meio de tais análises remetendo a mídia parâmetros para avaliação de veículos ${ }^{6}$.

Partindo do princípio de conceitos estatísticos, o presente estudo se baseia nas características descritas acima de parâmetros, entretanto este visa o controle do conforto acústico no interior de veículos SUV, levanto em consideração instrumentação, aquisição e tratamento estatístico de dados processados. Por meio deste princípio, foram utilizados métodos estatísticos descritivos combinados para abranger as variações de unidades e situações apresentadas.

\section{Metodologia}

\section{REFERENCIAL TEÓRICO}

O som é apenas uma vibração propagada transmitida em um meio (ar ou outro), e audível quando chega ao ouvido humano. A faixa do limiar auditivo de uma pessoa geralmente varia entre $20 \mathrm{~Hz}$ a $20 \mathrm{kHz}$, havendo mais sensibilidade na faixa de $100 \mathrm{~Hz}$ a $15 \mathrm{kHz}$. Vibrações são percebidas abaixo de $200 \mathrm{~Hz}$, podendo nesta faixa serem tanto sentidas quanto ouvidas. Pressão sonora acústica (P) serve de base nos estudos dos sons ${ }^{10}$. A faixa de pressão sonora audível está entre $2.10-5 \mathrm{~N} / \mathrm{m}^{2}<\mathrm{P}<20 \mathrm{~N} /$ $\mathrm{m}^{2}$. Geralmente não é prático utilizar esta faixa pois ela é suscetível a grandes variações. Sendo esta escala de manuseio complicado e não linear. Portanto a medida do nível de pressão sonora estudada será apresentada em decibel, uma vez que esta escala logarítmica se aproxima da percepção do ouvido às flutuações de pressão sonora ${ }^{11}$.

A escala bel é sem dimensão (relativa), e traz uma comparação quantitativa em relação à um valor referência, tomando como base limites inferiores ou superiores. A escala decibel possui algumas curvas e a que mais se adéqua ao projeto é a escala $\mathrm{dBA}$, comumente usada nas avaliações de ruído interno como apresentado na Figura 1 a seguir:

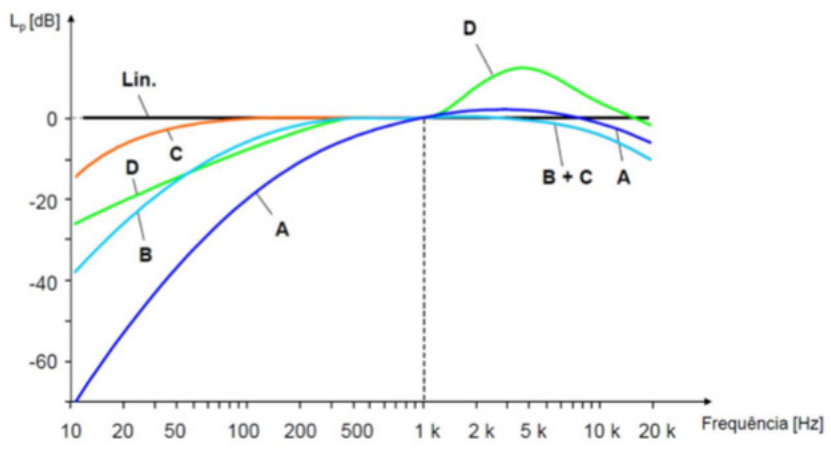

Figura 1: Escala dB. Fonte: ZAVALA ${ }^{12}$

Para Zavala, ruídos baixos a curva de ponderação acústica que melhor se aproxima da sensibilidade humana é a do tipo A, portanto é expressa como dBA ou $\mathrm{dB}(\mathrm{A})^{12}$.

Além da percepção acústica humana, também serão considerados os parâmetros de Articulation Index (\% Inelegibilidade humana) que se trata da quantidade que compreendemos dentro do nosso limiar auditivo em uma conversa. Os parâmetros de Articulation index também são considerados durante o tratamento de dados na escala $\mathrm{dBA}^{7}$.

Outro aspecto a se considerar é a análise de vibrações dentro de um veículo, uma vez que a quantidade de vibração (torcional ou não) produzida nos componentes veiculares influencia diretamente nas tratativas de problemas e até mesmo na decisão final do cliente. Oscilação (Vibração) pode ser explicada como qualquer movimento com repetição em determinado intervalo de tempo. Dentro das avaliações em uma medição internal noise (ruído interno) de NVH para veículos automotores, deve-se considerar a normativa ISO 2631 que traz formulações e parâmetros acerca da exposição humana de vibrações de corpo inteiro. Além destes parâmetros deve-se levar em consideração a exposição humana a vibrações transmitidas às mãos e aos braços contidos na ISO $5349^{13,14}$. 
Definir limites para emissões de ruído e vibração em veículos automotores no Brasil vêm sendo tratado por normativas como a NBR 15145 e a resolução do CONAMA $\mathrm{n}^{\circ} 1$, de 11 de fevereiro de 1993. Sendo que elas têm feito referência a ruído de passagem (Passbynoise) ${ }^{15,16}$.

\section{MATERIAIS}

Com o intuito de ter dados métricos, utilizou - se um padrão de equipamentos e configurações para gerar confiabilidade nas medições. A aquisição de dados com:

- 4 microfones (que possuem pré-amplificadores) de $1 / 2$ ” e flutuação sonora próxima ao limiar humano;

- 2 acelerômetros triaxiais (captando as ondulações em $\mathrm{X}, \mathrm{Y}$ e Z);

- 1 módulo para computar os dados;

- 1 tacógrafo para registro de dados rotacionais (RPM);

- O software signatureaquisition do conjunto simcenter testlab desenvolvido pela empresa Siemens.

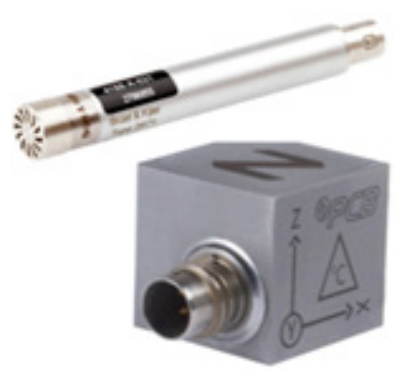

Figura 2: Microfone e acelerômetro. Fonte: SIEMENS ${ }^{17}$, PCB ${ }^{18}$

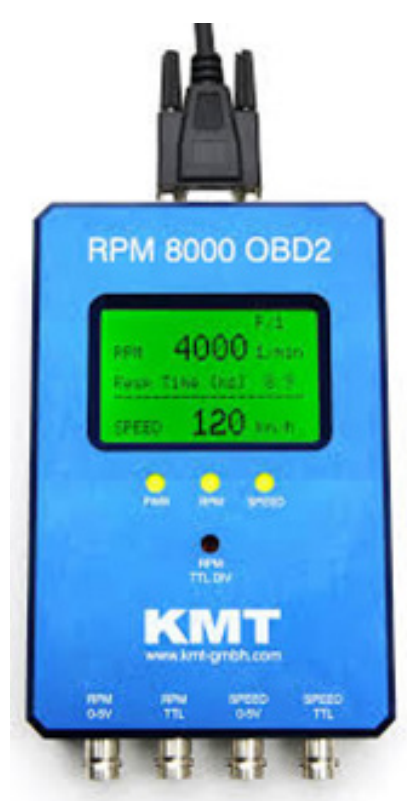

Figura 3: Speedsensor. Fonte: KMT ${ }^{20}$
O posicionamento dos microfones ajustado com a referência de 75 centímetros (verticalmente) do assento até a metade do encosto de cabeça do banco, partindo deste ponto mais 13 centímetros até a ponta do microfone no sentido horizontal, e da haste de suporte o microfone $8 \mathrm{~cm}$ para fora (evitando interferências com o suporte). Todos os microfones calibrados para 94dBA (configuração de fábrica).Os acelerômetros configurados com as fórmulas de ponderação contidas nas normativas ISO 2631 (SW12h - Steering Wheel at 12 hours posistion) e ISO 5349 (Dfost - Driver front outboardseat track). Sendo posicionados o Dfost no assoalho do veículo e o SW12h no volante para captar as vibrações de corpo inteiro e mãos e braços respectivamente. O tacógrafo conectado a entrada OBD do veículo e o módulo de aquisição de dados ${ }^{13,14,19}$.

\section{AQUISIÇÕES DE DADOS}

Este estudo avaliou os veículos em modo estacionário (na marcha drive) com o ar condicionado ligado, e em velocidade de cruzeiro. O teste de idle (conhecido como ocioso/ estacionário) é coletado relacionando tempo e ruído produzido. Durante 10 segundos será captada uma medição de insuflamento (com o ar ligado). Cinco medições são realizadas, objetivando prever possíveis descartes por adversidades externas ou configurações e ruídos anormais no veículo, em trecho de asfalto.

Em cruise (como o próprio nome diz "velocidade de cruzeiro") as medições realizadas em trecho de concreto a $120 \mathrm{~km} / \mathrm{h}$. Este também terá a aquisição de 5 medições em cada condição de velocidade, uma vez que as variações também serão consideradas nas análises das curvas de dBA por tempo.

\section{PROCESSAMENTOS DE DADOS}

Após mensurados os dados, e processados no software simcentertestlab 19.1 versão desktop advanced obteve se os SN (Single Numbers). Estes números baseiam-se nas corridas realizadas e nas ponderações por meio de filtros de cálculo das normativas ISO5349 e ISO2631. Com as médias energéticas (Single numbers) submetidas 
à análise sigma, considerando $k=1,5$ por se tratar de uma distribuição normal gaussiana os dados foram tratados utilizando o Sisvar ${ }^{13,14,21}$.

Análises de limiares produtivos vêm sendo produzidas pelo método seis sigma, considerando uma distribuição normal de variáveis. O CEP (Controle Estatístico do Processo) Este tipo de estudo estatístico se baseia no desvio padrão multiplicado por um fator $(\mathrm{K})$.

$$
\sigma=k * \text { desvpad }
$$

Entretanto considerando $\mathrm{K}$ como 3 ou 1,5 tem-se valores próximos ao limite superior da amostra estudada remetendo a uma distribuição gaussiana. Esta análise é comumente utilizada para delimitar limites do processo e gerenciamento da qualidade $22-24$. Ao partir da hipótese que a variável que se quer controlar segue uma distribuição normal, objetiva-se ter controle sobre o desvio padrão e a média. Caso não exista variação ao longo do tempo, tal dado pode ser caracterizado como um "processo sobre controle". Os dados aquisitados também seguiram o princípio da distribuição gaussiana, visando traçar o limite máximo para os parâmetros de ruído e vibração, partindo das seguintes equações: $:^{25-27}$.

\section{Limitemáx $=$ média $+\sigma$}

(Eq.02)

$\%$ devariaçãodaamostra $=\frac{\sigma}{\text { média }} \quad($ Eq.03)

Os cálculos de limites máximos foram baseados também na diferença significativa de Tukey. Método desenvolvido por John Tukey em 1953, embasado na comparação de variáveis, levando em consideração uma amostra normalizada de médias, partindo da atribuição da mínima diferença significativa e a amplitude estudentizada e não o percentual como no método sigma. Representado por:

$$
T S D=q \alpha \cdot(k, n-k) \cdot \sqrt{\frac{Q M E}{n}}
$$

A TSD (Tukey significant diference - Diferença significativa de Tukey) se baseia na amplitude estudentizada ( $\mathrm{G}$ ou k,k-n), no quadrado médio dos resíduos (QME) e número de replicas do nível (n).Para tamanhos amostrais balanceados, o teste de Tukey declara duas médias significativamente diferentes se o valor absoluto de suas diferenças não ultrapassar a amplitude (diferença do menor e maior valores mensurados neste comparativo), como representado por:

$$
Y \operatorname{máx}-Y \operatorname{mín}>T S D
$$

Partindo da correlação entre a igualdade da amostra e sua distribuição, os limites foram então calculados, visando ter uma amplitude estudentizada para suprir a variação dos valores de vibração nas condições propostas e parametrizar este valor para todos os modelos participantes do estudo.

\section{Resultados e Discussão}

Após mensurar todos os veículos até se alcançar uma quantidade para se obter confiabilidade nos resultados estatísticos, a amostra contou com 30 veículos de quatro modelos com diferentes versões, totalizando cinco medições de cada tipo de veículo. As marcas e modelos foram ocultadas e substituídas por letras a fim de resguardar o autor e a empresa parceira quanto a possíveis divulgações de informações reais. Os valores de ruído característico, obtidos (dBA) e vibração $\left(\mathrm{m} / \mathrm{s}^{2}\right)$ também foram ocultados dos resultados gráficos afim de mostrar neste apenas o comportamento da amostra, e também evidenciar a importância de correlacionar os métodos e a vantagem de ter utilizado o método de Tukey para tal parâmetro. Este limite tem como intuito servir de base para a correlação subjetiva.

Os padrões analisados para este estudo estatístico se baseiam na análise de desvio padrão e escala três sigma, comparada com o método de Tukey. Trazendo para os 
dados acústicos as métricas por meio do software Sisvar.

A necessidade de adotar um método estatístico auxiliar se deu devido à variação de amplitude da amostra ao considerar dados de vibração no assoalho e no volante, uma vez que há uma considerável diferença entre ambos. Este comportamento de vibração em assoalho (Dfost) é bem característico e como esperado maior se comparado a vibração em mãos e braços - Volante (SW12H), uma vez que adotada a amplitude estudentizada baseada na variação do desvio padrão amostral.

Já para os dados de ruído interno, os dois métodos tiveram resultados positivos, uma vez que a análise sigma apenas consideraria a diferença amostral e traria bons resultados para este parâmetro logaritmo. Entretanto, os valores foram parametrizados através da adoção da melhor amplitude estudentizada pelo método de Tukey. Para ambos os valores característicos o método considerou o descarte de corridas que estivessem fora do percentual, fazendo com que os veículos além de serem da mesma classe só seriam válidos para o projeto caso nenhuma intempérie interferisse nas corridas, como ressonância, deterioração e velocidade do vento alta.

O teste de Tukey pôde ser apresentado nas linhas gráficas abaixo, remetendo a diferença do limite definido utilizando uma amostra apenas pelo método três sigma, e pela análise sigma combinada com a comparação de médias de Tukey.

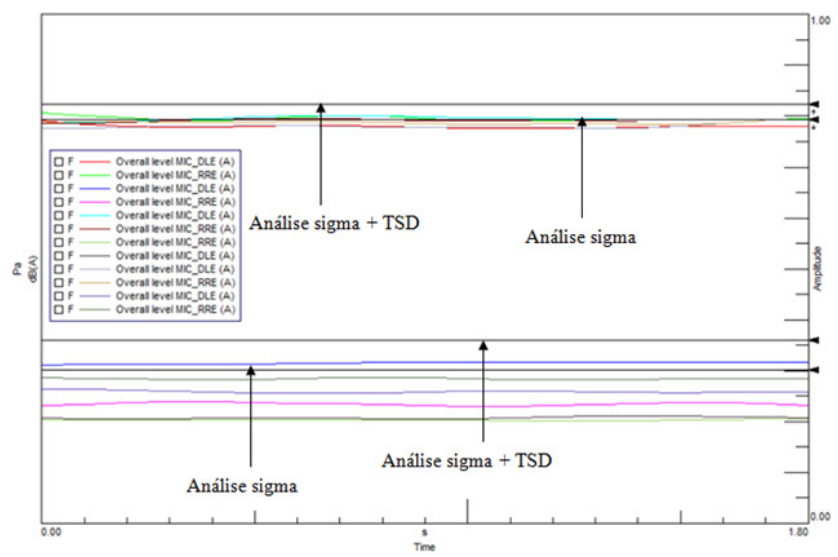

Gráfico 1: Resultados obtidos em dispersão para pressão sonora. Fonte: Autor
No Gráfico 1 nota-se que o comportamento inferior (ruído mais baixo) se refere aos testes de idle, enquanto que as linhas na parte superior do gráfico se referem ao teste de Cruise. Partindo da dispersão apresentada pelas medições, infere-se que ao utilizar apenas a análise sigma para este parâmetro os resultados seriam satisfatórios, porém próximos aos valores mensurados. A linha superior em ambos os casos se trata do resultado utilizando o método de Tukey, como observado não detendo grande variação e atendendo aos parâmetros dos quatro modelos mensurados.

No Gráfico 2 tem-se uma amostra da diferença em se tratar dados utilizando apenas o método três sigma, e ao correlacionar tais parâmetros com o método de John Tukey. Ao utilizar apenas a análise sigma em alguns pontos o LMS (Limite máximo superior) tange a linha mensurada, remetendo a um limite dentro da amostra. Seguindo esta ideologia a abrangência da utilização de métodos combinados foi proporcional tanto em ensaios de ruído quanto e dados de vibração (Gráfico 3).

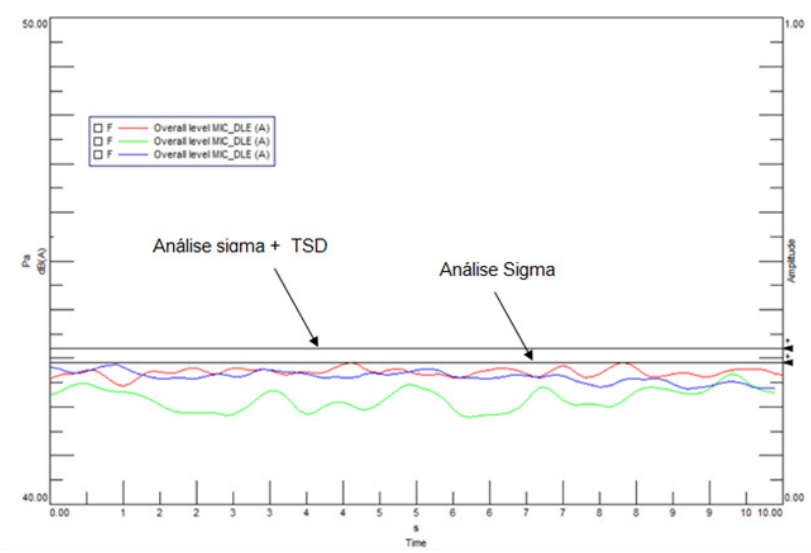

Gráfico 2: Correlação para ruído de teste em modo estacionário dos dados estatísticos. Fonte: Autor

Os parâmetros demonstrados representativamente a seguir trazem à tona a principal contribuição da análise realizada, uma vez que permeou a representação e o cálculo 
independente do modelo, ano ou mesmo nota acústica, pois tal correlação depende somente da amplitude estudentizada e análise de desvio padrão para classificação amostral.

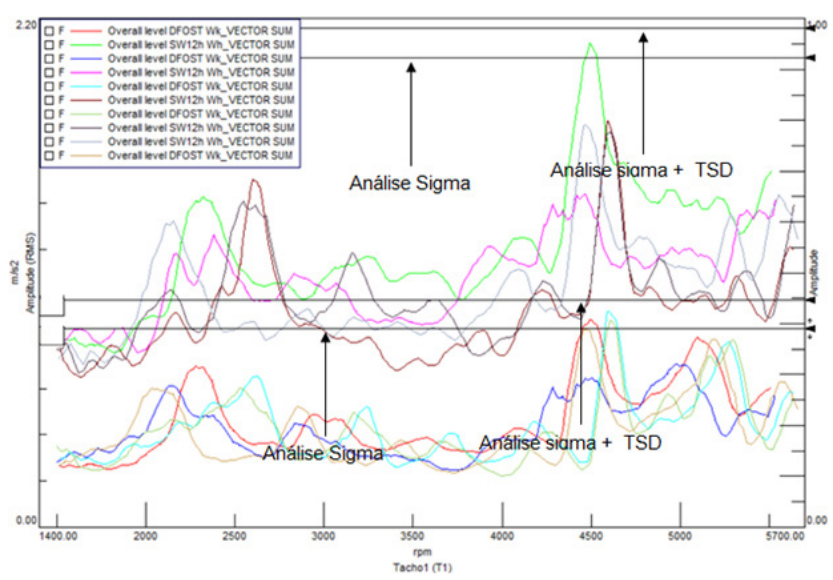

Gráfico 3: Correlação para vibração em corpo inteiro e mãos e braços. Fonte: Autor

\section{Conclusões}

O método de Tukey é constantemente utilizado no meio acadêmico, entretanto para o ambiente industrial a análise de limites por meio do cálculo sigma é mais modesto seu emprego. Com a padronizaçãoda análise de ruído interno, além de sanar reclamações de campo, contribuiu-se com ocontrole de qualidade, uma vez que ao se obter um estudo de $\mathrm{NVH}$, frequentemente a qualidade acústica do veículo estará sendo aprimorada. Portanto, para o nosso teste, com variáveis balanceadas (em mesma quantidade), o método de Tukey foi adequado para realizar estas comparações entre médias, levando em consideração que temos dados em vários parâmetros, o que acarretaria testes percentuais diferentes.

O método é utilizado para testar toda e qualquer diferença entre duas médias de tratamento, comumente aplicado em tratamento de ANOVA (Análise de variância) por ter como base a DMS (diferença mínima significativa), além de poder ser utilizado aliado a outros métodos como no referido experimento.
Ações de benchmarking tem levado em consideração tais estudos, visando aumentar a confiabilidade e a qualidade do produto final. Partindo desta premissa o controle acústico e a análise estatística confrontando dados é uma prática imprescindível de NVH.

Deste modo conclui-se que para os dados de vibração, a padronização da amplitude estudentizada utilizada no método de Tukey gerou uma unificação nos limites, fazendo com que as variações de modelo e versões de veículos, dentro de cada categoria avaliada fossem atendidas. A aplicação da dispersão amostral foi satisfatória para avaliação do ruído interno, fazendo com que os limites (embora não demonstrados numericamente neste) possibilitassem utilizar apenas o método três sigma ou a combinação de ambos.

\section{Referências}

1. PEREIRA.Rayann .Importância da probabilidade e estatística para os profissionais de engenharia civil. Disponívelem: $<$ http://anais. unievangelica.edu.br/index.php/cifaeg/article/view/572>. Acesso em: 15 Nov.2019

2. TOLEDO, Geraldo Luciano. OVALLE, Ivo Izidoro. Estatística Básica. 2 ed. São Paulo: Atlas, 1995.

3. WERKEMA, M.C.C. Ferramentas estatísticas básicas para o gerenciamento de processos. Belo Horizonte: Fundação Christiano Ottoni, 2006.

4. CAMPOS, Vicente F. TQC: Controle da Qualidade Total (no estilo Japonês). Rio de Janeiro: Bloch Ed, 2004.

5. CORDEIRO,GaussM.Históriadaestatística.Disponívelem:<file://C:/ Users/User/Downloads/hist \% C 3\% B 3 ria \% 20 da $\% 20$ estat\%C3\%ADstica.pdf>.Acesso em: 11 Nov. 2019

6. SURIANO, Márcia Thais. Ferramenta de apoio à decisão para o controle da poluição sonora urbana Disponível em: $<$ http://www. scielo.br/pdf/csc/v20n7/1413-8123-csc-20-07-2201.pdf >.Acesso em:15 Nov. 2019.

7. MCDERMOTT, J. Sound and the Ear, MIT OCW, 2004

8. ABDEL-RAHMAN, E.M.; NAYFEH, A.H.; ZIYAD N.M. Dynamics and Control of Cranes: A Review, A Review Journal of Vibration and Control; 9: 863 - 908, 2003.

9. MAFFRA, Sérgio Alvares Rodrigues de Souza. Propagação de som em ambientes acústicos virtuais bidimensionais. Disponível em: $<$ https://www.maxwell.vrac.puc-rio.br/3723/3723_1.PDF $>$. Acesso em: 25 Ago. 2019. 
10. PERCON. Teste NVH. Disponível em: < www.acessopercon.com>. Acesso em: 08 de Abr. de 2019

11. FAU. Db, Pressão, Potência e intensidade. Disponível em: < http://www.fau.usp.br/arquivos/disciplinas/au/aut0278/Aulas/ AUT0278\%20Aula\%2004\%20-\%20dB\%20Press\%C3\%A3o\%20 Pot $\%$ C3\%AAncia\%20Intensidade.pdf $>$.Acesso em: 22 de Abr. de 2019.

12. ZAVALA, Paulo. Treinamento em avaliação e controle de ruído e vibração veicular. Anápolis - GO. Agosto de 2018.

13. INTERNATIONAL ORGANIZATION FOR STANDARDIZATION (ISO). ISO2631: Guide for evaluation of human exposure to whole body vibration. United States. 1978

14. INTERNATIONAL ORGANIZATION FOR STANDARDIZATION (ISO). ISO5349: Guide for evaluation of human exposure to hands vibration. United States. 1979.

15. ASSOCIAÇÃO BRASILEIRA DE NORMAS TÉCNICAS (ABNT). NBR15145: Acústica: Medição de ruído emitido por veículos rodoviários automotores em aceleração: Método de engenharia. Rio de Janeiro 2004.

16. RES, D. E.; Perigosos, P. Resolução CONAMA no 001, de 11 de fevereiro de 1993 Publicada no DOU n. , p. 495-498, 1993.

17. BRUEL\&KJAER. Desenvolvimento de ruído, vibração e aspereza(NVH) em veículos. Disponível em: <https://www.bksv. $\mathrm{com} / \mathrm{ptBR} /$ Applications/vehicle-noise-vibration-and-harshnessdevelopment>. Acesso em: 09 de Abr. de 2019.

18. PCB. Acelerômetro triaxial. Disponível em: $<$ https://i.ytimg.com/ vi/2w3A9Az9u0k/maxresdefault.jpg >. Acesso em : 03 Nov. 2019.

19. SIEMENS.Simcentertestlab.Disponívelem: $<$ https://www.plm. automation.siemens.com/global/pt/products/simcenter/testlab.html $>$. Acesso em: 13 de Mai. de 2019.

20. KMT.Speedsensor.Disponívelem: $<$ https://www.kmttelemetry.com/ fileadmin/media/Downloads/SpeedRPM/RPM-8000-OBD2-UM.pdf - manual>.Acesso em: 12 Nov. 2019.

21. Ferreira, D. F. Sisvar: a Guide for its Bootstrap procedures in multiple comparisons. Ciência e Agrotecnologia, v. 38, n. 2, p. 109-112, 2014. Disponível em: $<$ http://www.scielo.br/scielo.php?script $=$ sci arttext\&pid $=$ S1413-70542014000200001\&lng $=$ en\&tlng $=$ en $>$. Acesso em: 22 Mai. 2019.

22. Aguiar, S. Integração das ferramentas da qualidade ao PDCA e ao programa seis sigma. Editora de Desenvolvimento Gerencial Belo Horizonte, 2002.

23. Fernandes, M. M. Análise doprocesso de seleção de projetos seis sigma em empresas de manufatura no Brasil. Universidade Federal de Itajubá, 2006.

24. Nortegubisian.Controle estatístico do processo. Disponível em: $<$ https://www.nortegubisian.com.br/blog/controle-estatistico-doprocesso-cep>. Acesso em: 09 de Out. 2019

25. Slack, Nigel. Administração de Produção. São Paulo: Atlas, 2002

26. Martins, Petrônio G.; Laugeni. Fernando P. Administração da Produção. São Paulo : Saraiva, $5^{\mathrm{a}}$ Ed., 2005.

27. Blog da Qualidade. Controle estatístico de processo. Disponível em: $<$ https://blogdaqualidade.com.br/controle-estatístico-de-processocep/>. Acesso em: 09 de out. 2019

\section{Lays E. Cunha* \& Fábio S. Gomes}

Centro Universitário de Anápolis - UniEVANGÉLICA. Avenida Universitária, km 3,5, Centro Universitário, Anápolis, Goiás, Brasil.

*E-mail: lays1207@hotmail.com 